\title{
Three-Dimensional (3D) Anthropometry Study of the Malaysian Population
}

\author{
Yii Bonn BONG*1 ${ }^{*}$ Amir F. MERICAN ${ }^{1,2}$, Suhaila AZHAR ${ }^{1}$, Tahereh MOKHTARI ${ }^{1}$, \\ Abdul Majid MOHAMED ${ }^{1,3}$, Asma A. SHARIFF ${ }^{1,3}$ \\ ${ }^{1}$ Centre of Research for Computational Sciences and Informatics in Biology, Bio industry, Environment, \\ Agriculture and Healthcare, University of Malaya, Kuala Lumpur, Malaysia; \\ ${ }^{2}$ Institute of Biological Sciences, Faculty of Science, University of Malaya, Kuala Lumpur, Malaysia; \\ ${ }^{3}$ Centre for Foundation Studies in Science, University of Malaya, Kuala Lumpur, Malaysia
}

http://dx.doi.org/10.15221/14.167

\begin{abstract}
Three-dimensional (3D) anthropometry has become an emerging field with the advancement of 3D body scanning technology over the recent years. Conventional method of anthropometry data measurement is time consuming and error-prone. The 3D whole-body surface scanner, which was initially developed for the clothing industry, contributes to healthcare application by providing accurate anthropometric measurements and data visualization in body size, shape and skin surface area. The body scanner utilized 3D photogrammetry technology to generate high quality digital anthropometric information about shapes and sizes of an individual. The aim of the study is to investigate body size and shape of Malaysian females. A cross-sectional study was conducted using random sampling technique. Recruitment of subjects were randomized and based on age and ethnicity. A preliminary study of 160 female subjects was carried out. In addition to the scanning procedures using the body scanner, selected manual anthropometric measurements for height, weight and skinfold thickness were obtained using manual methods and demographic data such as age and ethnic groups were recorded. All manual measurement followed the requirements and procedures stated in the respective ISO documents, involving body posture, landmark and the instrument used. Descriptive statistical analysis for body dimensions and calculations were carried out. The 3D scanners undoubtedly produced highly accurate, consistent and repeatable measurements. However, the scan data can be slightly different from traditional anthropometric data especially for circumference measurements such as chest, waist and hip. Therefore, scanned anthropometric data and manual measurement data are more useful together. We envisage that this study would be beneficial for healthcare providers for growth monitoring and early diagnosis of health problems related to obesity especially for the Malaysian population. Although the study focuses on the healthcare sector, datasets can also be utilized by the industry in the design and production of consumer products related on the human body.
\end{abstract}

Keywords: 3D anthropometry, phenotypic variation, Malaysian population, 3D body scanner, manual anthropometry measurement

\section{Introduction}

Anthropometry is the scientific study of variation in the size and shape of the human body. Anthropometric data refers to a collection of physical dimensions of the human body. It plays an important role in the field of industrial design, clothing, ergonomics and healthcare. It is also used as a tool to estimate the nutritional status of a population as well as to monitor the health and growth of an individual. Conventional methods of manually measuring thousands of individuals to obtain statistically useful measurement data are time-consuming, error-prone and expensive. As such, the 3D whole body scanner was utilized to obtain high quality digital anthropometric information about shapes and sizes of individuals.

Traditional anthropometry largely relies on expert use of tapes and calipers that are generally limited to single dimensional (1D) information to describe the human body. Body scanning technology, on the other hand, provides 3D data of whole body surface, thus the measurement capabilities can be directly extended to body volume and surface areas. The scanner is more precise and consistent than even trained human beings, requiring 8-9 seconds per stance to capture the needed data [1]. 3D body scanning technology provides measuring capabilities for segmental volumes and surface areas, and for large surveys which are now less expensive, faster, and more reliable.

*yiibonn@um.edu.my / yiibonn@gmail.com; +6012-804-1666 
Digital body scanning provides accurate measurements that are obtained in a digital format and can be integrated automatically into the computer without human intervention, a process which takes additional time and may introduce error. The data in 3D image form would be easier to analyze and classify into the three main body shapes of human physique: endomorph, mesomorph and ectomorph.

3D digital body scanner has found many diverse applications in industry, healthcare and other areas [2]. 3D scanning offers a technique to capture the body dimension in fast and reproducible way. One of the most important benefits is that the data of the subject can be stored on disks and retrieved when necessary. The technology for producing 3D coordinates for accurate computer representation of a scanned whole human body is currently being refined for practical use. These technologies are useful to the apparel industry in the potential availability of better fit models for consumer products and greater availability of made-to-measure or custom garment at an affordable price [3]. In healthcare, anthropometric measurements for children reflects the general health status, dietary deficiency as well as growth and development over time. In addition, adult anthropometric data is used for evaluation of health and dietary status, disease risk and body composition changes over time [4]. For instance, arm circumference is used to classify the nutritional risk and/or the status of an individual throughout the process of aging in terms of physiological and nutritional changes [5].

National sizing surveys using 3D whole body scanners were successfully conducted in the UK and USA (SizeUK and SizeUSA, respectively). Similar surveys were also conducted in France, Thailand, Korea, Mexico and Australia [6, 7]. The Malaysian Sizing Survey (MySIZE) is a national anthropometric study of the physical body sizes and shapes of Malaysians utilizing 3D body scanner and the traditional anthropometric measurements. Its aims are to create national database containing digital anthropometric data of body size and shape as well as to contribute to worldwide data gathering initiative pertaining to body size and shape for current and future advancement in human lifestyle, healthcare and medical treatment.

As such, this preliminary study is conducted to investigate the body size and shape of Malaysian females. Besides, the difference between scanned measurement and manual measurements were compared. Due to the lack of sizing surveys in the country, the US and / or UK size charts were used by local retailers and manufacturers. However, the anthropometric data for these charts were from a population very different from that of Malaysia. As a result, the manufactured products might not fit the local body types. Consumers are often forced to have alterations done or simply wearing ill-fitted clothing. Sometimes, the size charts from other countries are adapted and modified based on the manufacturers' instincts and experiences to describe the Malaysian body [8]. Consequently, each brand has its own sizing label which may be very different from those of the other brand. This variation in sizing systems between brands leads to confusion among consumers.

\section{Method}

\subsection{Subjects and Participation}

A cross-sectional study was conducted among Malaysian females aged 18 to 65 years old. The subjects were recruited at random based on age and ethnicity. Ethnic differences in growth were considered superficially in this project. Hence, the three major ethnic groups (Malays, Chinese and Indian) and other indigenous groups were included. Subjects were briefed and asked to sign consent forms before participation to assure confidentiality.

\subsection{Manual Measurements}

Manual anthropometric measurements of the subjects were taken after they had changed into tight-fitting scan wear (see section 2.3). The actual body measurements of height, weight, skinfold measurement and circumference measurements were taken. All body measurements were taken on the right side of the body by the same trained measurer twice to check for intra-observer error. Body weight was measured using the Omron HBF 514C body composition monitor (Omron Healthcare Inc., USA) and scale while the height of the subjects were measured using the Seca body meter (Seca, Germany).

For height and weight measurements, subjects were required to stand straight on the floor board of the body meter with their backs to the vertical backboard of the body meter scale and their head maintained in the Frankfort Horizontal Plane position. The heels of the feet were placed together with both heels touching the base of the vertical board. The subjects were asked to inhale deeply and to 
stand fully erect without altering the position of the heels. Height was recorded to the nearest $0.1 \mathrm{~cm}$. For weight, the subjects were asked to stand on the center of the weight scale platform with their weight evenly distributed on both feet. Weight was recorded to the nearest $0.1 \mathrm{~kg}$.

Circumference measurements were taken to the nearest $0.1 \mathrm{~cm}$ using body tape. For arm circumference measurement, subjects were asked to stand with their elbows relaxed and their right arms hung freely to the side. The measuring tape was placed around the upper arm at the marked point. When measuring the waist circumference, subjects were asked to lift their cloths to expose the waist area. The measurer stood behind the subject and palpated the hip area for the right iliac crest and marked a horizontal line at the high point of the iliac crest and then crosses the line to indicate the mid-axillary line of the body. The measurement was made to the nearest $0.1 \mathrm{~cm}$ at minimal respiration. The hip measurements were taken with the subjects standing erect with feet together and weight evenly distributed on both feet. The body tape was then placed at the maximum extension of the buttocks. The measurer then adjusted the sides of the tape and checked the front and sides so as the plane of the tape is horizontal. For thigh circumference, subjects were asked to stand with the right leg in front of the left leg and the body weight shifted back to the left leg. The measuring tape was placed around the mid-thigh and measurement was taken to the nearest $0.1 \mathrm{~cm}$.

All skinfolds were measured with the Holtain skinfold calipers (Holtain Ltd, UK). The measurer used her left thumb and forefinger and gently grasped the fold of skin and underlying subcutaneous adipose tissue. The amount grasped depended upon the thickness of the subcutaneous adipose tissue. The measurer grasped enough skin and adipose tissue to form a distinct fold that separates from the underlying muscle. The jaws of the calipers were placed at the marked level, perpendicular to the length of the fold, and the skinfold thickness was measured to the nearest $0.1 \mathrm{~mm}$.

\subsection{D Whole Body Scanner Automatic Measurements}

The $\mathrm{TC}^{2} \mathrm{NX}-16$ body scanner (TC ${ }^{2}$, USA) was used in this study. This 3D whole body scanner is non-invasive which projects strips of safe 'white light' onto the body form and records distortions by using non-moving cameras. These lights are not skin-deep intrusive and non-contact. Subjects were asked to change into a specially-designed scan wear made from Lycra material which were available in five sizes of extra-small (XS), small (S), medium (M), large (L) and extra-large (XL). They then adopted the standardized standing position and held the stabilizing handholds to maintain these correct positions during scanning.

The Body Measurement System Software [ver.5.3, TC ${ }^{2}$, USA] was used for the purpose of analysis. The software automatically extracts about 200 measurements from each scan. All automatic measurements using both the ISO 8559 and SizeUSA references were generated from the software.

\subsection{Statistical Analysis}

Basic descriptive statistics for body dimensions were calculated to determine the characteristics and measurements of the subjects. The Statistical Package for the Social Science (IBM SPSS Statistics Ver.22, IBM Corp.) was used for data entry and descriptive statistical analysis. The differences between manual and body scanner measurements were compared using paired sample t-test analysis.

\subsection{Malaysian 3D Anthropometric Database}

All manual and automated datasets from this study were stored in a specially designed database called the Malaysian 3D Anthropometric Database. Database provides a well management asset in managing complex data [9] and is able to integrate the information and source which create powerful searches [10]. The advantages of using database included reduction in data redundancy, data duplication and ease of information searching. The system is able to provide a medium of interaction between the database and the user (graphical user interface, GUI) for records management, information storage and information display. 


\section{Results and Discussions}

\subsection{Subject's Demographic Characteristics}

The demographic characteristics of the studied subjects are shown in Table 1. A total of 160 female subjects were obtained for the preliminary study. The mean and standard deviation of age was $27.9 \pm$ 8.8 years old. Subjects were mostly in the $\leq 20$ years old and $20-30$ years old age groups $(75.0 \%)$ while a quarter of the sample was in the age ranging from 30 to $\geq 40$ years old. The distribution of ethnicity was $75.6 \%$ Malays, $21.3 \%$ Chinese, $2.5 \%$ Indians and less than $1 \%$ of other indigenous groups. In this study, a majority of the subjects were students $(121,75.6 \%)$ while the remainder were working adults.

Table 1. Demographic characteristics of the subjects.

\begin{tabular}{|l|l|}
\hline \multicolumn{1}{|c|}{ Demographic characteristic } & Number of subjects (\%) \\
\hline Age groups & \\
$\leq 20$ years old & $8(5.0)$ \\
$20-30$ years old & $112(70.0)$ \\
$30-40$ years old & $22(13.75)$ \\
$\geq 40$ years old & $18(11.25)$ \\
Mean Age \pm SD 27.9 \pm 8.8 & \\
\hline Race & \\
Malay & $121(75.6)$ \\
Chinese & $34(21.3)$ \\
Indian & $4(2.5)$ \\
Other indigenous groups & $1(0.6)$ \\
\hline Occupation & $121(75.6)$ \\
Student & $39(24.4)$ \\
Employed &
\end{tabular}

The means and standard deviations for manual anthropometry measurements are shown in Table 2.

Table 2. Means and standard deviations for manual measurements.

\begin{tabular}{|l|l|}
\hline \multicolumn{1}{|c|}{ Parameters } & \multicolumn{1}{|c|}{ Manual measurements (Mean \pm SD) } \\
\hline (1) Body height (in $\mathrm{cm}$ ) & $156.00 \pm 5.00$ \\
(2) Body weight (in kg) & $60.10 \pm 16.08$ \\
(3) Body Mass Index & $24.55 \pm 6.03$ \\
(4) Visceral fat & $4.93 \pm 2.53$ \\
(5) Body fat percentage (\%) & $35.37 \pm 10.00$ \\
(6) Skeletal muscle percentage (\%) & $26.03 \pm 3.32$ \\
(7) Skinfold measurements (in $\mathrm{mm})$ & \\
- Triceps & $28.72 \pm 7.53$ \\
- Abdominal & $29.50 \pm 12.89$ \\
- Thigh & $42.57 \pm 12.15$ \\
\hline
\end{tabular}

The consistency of 3D body scanner-extracted measurements were validated with those manually measured dimensions, especially the circumference parameter. It is necessary to examine the differences and compatibility between 3D scan measurements and manual measurements. Thus, the comparison of mean and standard deviation for circumference measurements from manual and those obtained from scanner are presented in Table 3. Both SizeUSA and ISO 8559 references for the body scanner measurements were included for the purpose of comparison. 
Table 3. Comparison of means and standard deviations for circumference of the body dimensions from manual measurements and body scanner measurements (SizeUSA reference and ISO 8559 reference).

\begin{tabular}{|l|l|l|l|}
\hline \multirow{2}{*}{ Parameters } & $\begin{array}{l}\text { Manual } \\
\text { measurements } \\
\end{array}$ & Body scanner measurements (Mean \pm SD) \\
\cline { 3 - 4 } & Mean \pm SD) & SizeUSA reference & $\begin{array}{r}\text { ISO } 8559 \\
\text { size reference }\end{array}$ \\
\hline Neck & $35.27 \pm 4.40$ & $35.40 \pm 7.48$ & $36.45 \pm 7.06$ \\
\hline Arm & $30.50 \pm 6.06$ & $30.01 \pm 5.13$ & $30.37 \pm 6.19$ \\
\hline Chest & $90.45 \pm 11.11$ & $90.82 \pm 10.07$ & $92.40 \pm 12.21$ \\
\hline Waist & $83.09 \pm 14.42$ & $83.00 \pm 12.42$ & $83.06 \pm 12.58$ \\
\hline Hip & $91.80 \pm 14.34$ & $90.41 \pm 14.34$ & $101.52 \pm 11.35$ \\
\hline Thigh & $56.06 \pm 7.78$ & $56.67 \pm 9.23$ & $60.95 \pm 9.16$ \\
\hline
\end{tabular}

Hip circumference measurement taken manually and using the body scanner according to the ISO 8559 size reference showed large difference. Paired t-test confirmed this hypothesis (Table 4). The mean \pm standard deviations of SizeUSA reference from 3D body scanner were found higher than the manual measurements for neck, chest and thigh circumferences. However, the manual measurements were found to have significant differences with the ISO 8559 size reference for neck, chest, hip and thigh circumferences. From the paired t-test analysis, measurements from the body scanner following USA size reference shows better and more compatible results than the ISO 8559 size reference.

Table 4. Paired t-test analysis for manual and body scanner measurements.

\begin{tabular}{|c|c|c|c|c|c|}
\hline Pair & $\begin{array}{l}\text { Circumference } \\
\text { measurement }\end{array}$ & Mean \pm SD & $t$ & $p$ value & Interpretation \\
\hline & $\begin{array}{l}\text { Manual vs SizeUSA } \\
\text { reference }\end{array}$ & & & & \\
\hline Pair 1 & $\begin{array}{l}\text { Neck - manual } \\
\text { Neck - SizeUSA }\end{array}$ & $\begin{array}{l}35.30 \pm 4.42 \\
35.40 \pm 7.48\end{array}$ & -0.016 & 0.86 & n.s. \\
\hline Pair 2 & $\begin{array}{l}\text { Arm - manual } \\
\text { Arm - SizeUSA }\end{array}$ & $\begin{array}{l}30.50 \pm 6.06 \\
30.01 \pm 5.13\end{array}$ & 1.27 & 0.20 & n.s. \\
\hline Pair 3 & $\begin{array}{l}\text { Chest - manual } \\
\text { Chest - SizeUSA }\end{array}$ & $\begin{array}{l}90.45 \pm 11.11 \\
90.82 \pm 10.07\end{array}$ & -1.13 & 0.25 & n.s. \\
\hline Pair 4 & $\begin{array}{l}\text { Waist - manual } \\
\text { Waist - SizeUSA }\end{array}$ & $\begin{array}{l}83.09 \pm 14.42 \\
83.00 \pm 12.42\end{array}$ & 0.19 & 0.85 & n.s. \\
\hline Pair 5 & $\begin{array}{l}\text { Hip - manual } \\
\text { Hip-SizeUSA }\end{array}$ & $\begin{array}{l}91.80 \pm 14.34 \\
90.41 \pm 14.34\end{array}$ & 4.12 & 0.000 & $* *$ \\
\hline Pair 6 & $\begin{array}{l}\text { Thigh - manual } \\
\text { Thigh - SizeUSA }\end{array}$ & $\begin{array}{l}56.06 \pm 7.78 \\
56.67 \pm 9.23 \\
\end{array}$ & -1.80 & 0.07 & n.s. \\
\hline & $\begin{array}{l}\text { Manual vs ISO } 8559 \\
\text { size reference }\end{array}$ & & & & \\
\hline Pair 1 & $\begin{array}{l}\text { Neck - manual } \\
\text { Neck - ISO } 8559\end{array}$ & $\begin{array}{l}35.30 \pm 4.42 \\
36.45 \pm 7.06\end{array}$ & -1.93 & 0.05 & * \\
\hline Pair 2 & $\begin{array}{l}\text { Arm - manual } \\
\text { Arm - ISO } 8559\end{array}$ & $\begin{array}{l}30.50 \pm 6.06 \\
30.37 \pm 6.19\end{array}$ & 0.29 & 0.76 & n.s. \\
\hline Pair 3 & $\begin{array}{l}\text { Chest - manual } \\
\text { Chest - ISO } 8559\end{array}$ & $\begin{array}{l}90.45 \pm 11.11 \\
92.40 \pm 12.21\end{array}$ & -4.49 & 0.001 & ** \\
\hline Pair 4 & $\begin{array}{l}\text { Waist - manual } \\
\text { Waist - ISO } 8559\end{array}$ & $\begin{array}{l}83.09 \pm 14.42 \\
83.06 \pm 12.58\end{array}$ & 0.06 & 0.94 & n.s. \\
\hline Pair 5 & $\begin{array}{l}\text { Hip - manual } \\
\text { Hip- ISO } 8559\end{array}$ & $\begin{array}{l}91.80 \pm 14.34 \\
101.52 \pm 11.35\end{array}$ & -18.49 & 0.000 & $* *$ \\
\hline Pair 6 & $\begin{array}{l}\text { Thigh - manual } \\
\text { Thigh - ISO } 8559\end{array}$ & $\begin{array}{l}56.06 \pm 7.78 \\
60.95 \pm 9.16\end{array}$ & -14.61 & 0.000 & $* *$ \\
\hline
\end{tabular}

${ }^{* *} p<0.01$

${ }^{*} p<0.05$

n.s. non-significant 
The 3D body scanner produced higher readings in some of the circumference measurements as compared to the manual measurements (Table 4). A possible explanation is that the scanner captured fat tissue underneath the skin and considered them as part of the body dimension. The human body is elastic in nature, whereby body size will decrease when the body tape was used in manual measurement. It is, therefore, important to conduct additional manual measurement to rule out all possible factors affecting the scan readings.

The means and standard deviations for selected circumference measurements from the present study as compared with that of the SizeUK and SizeThai are presented in Table 5. All three studies used the same scanning protocol. The Asian females have smaller measurements for chest, waist and hip than their UK counterparts. However, since the present study was preliminary, the results cannot be generalized to the whole Malaysian female population. More data is needed for a detailed analysis.

Table 5. Means and standard deviations comparison for circumference of the body dimensions from the present study with SizeUK and Size Thai ${ }^{[11]}$.

\begin{tabular}{|l|l|l|l|}
\hline Parameters & $\begin{array}{l}\text { Present study, } \mathrm{N}=160 \\
(\text { Mean } \pm \text { SD) }\end{array}$ & $\begin{array}{l}\text { SizeUK } \\
(\text { Mean } \pm \text { SD) }\end{array}$ & $\begin{array}{l}\text { SizeThai }^{[1] 1}, \mathrm{~N}=6499 \\
(\text { Mean } \pm \text { SD) }\end{array}$ \\
\hline Arm & $30.01 \pm 5.13$ & $28.7 \pm 4.1$ & $26.9 \pm 3.6$ \\
\hline Chest & $90.82 \pm 10.07$ & $94.7 \pm 8.6$ & $90.7 \pm 7.7$ \\
\hline Waist & $83.00 \pm 12.42$ & $86.9 \pm 11.4$ & $79.3 \pm 9.5$ \\
\hline Hip & $90.41 \pm 14.34$ & $103.8 \pm 9.7$ & $95.9 \pm 7.3$ \\
\hline Thigh & $56.67 \pm 9.23$ & $49.2 \pm 4.8$ & $46.9 \pm 4.4$ \\
\hline
\end{tabular}

\section{Conclusions}

The technology of producing 3D coordinates for accurate computer representation is currently being refined for practical use of scanned human body. In healthcare, anthropometric measurement for children reflect the general health status, dietary deficiency as well as growth and development over time. Adult anthropometric data is used for evaluation of health and dietary status, disease risk and body composition changes over time. The 3D scanners undoubtedly produced highly accurate, consistent and repeatable measurements; however, the scan data can be slightly different from traditional anthropometric data especially for circumferences such as chest, waist and hip. It is necessary to know clearly and precisely how and where measurements were taken. Therefore, scanned anthropometric data and manual measurement data are more useful together.

\section{Acknowledgements}

We would like to thank University of Malaya for funding this study (UMRG Program RP004-13AFR). We thank the research assistants for their help with the study.

\section{References}

[1] P. Treleaven, "Sizing Us Up", in IEEE Spectrum, Vol.41, No.4, 2004, pp. 28-31, http://dx.doi.org/10.1109/MSPEC.2004.1279190.

[2] T. Jing et al, "Scanning 3D Full Human Bodies using Kinects", in Visualization and Computer Graphics, IEEE Transactions on, Vol.18, Issue 4, 2012, pp.643-650, http://dx.doi.org/10.1109/TVCG.2012.56.

[3] R. P. Pargas, N. J. Staples and J. S. Davis, "Automatic measurement extraction for apparel from a three-dimensional body scan", in Optics and Lasers in Engineering, Vol.28, Issue 2, 1997, pp. 157-172, http://dx.doi.org/10.1016/S0143-8166(97)00009-2.

[4] C. D. Fryar, Q. Gu and C. L. Ogden, "Anthropometric Reference Data for Children and Adults: United States, 2007-2010", in Vital Health Stat, Vol.11, No.252, 2012 , http://www.cdc.gov/nchs/data/series/sr 11/sr11 252.pdf., accessed 2014.

[5] Z. Milanovic et al, "Basic Anthropometry and Body Composition Characteristic in an Elderly Population: A Systemic Review", in Physical Education and Sport, Vol.9, No.2, 2011, pp.173-182, http://facta.junis.ni.ac.rs/pe/pe201102/pe201102-07.pdf., accessed 2014. 
[6] P. Treleaven and J. Wells, "3D Body Scanning and Healthcare Applications", in IEEE Computer Society, Vol.40, No.7, 2007, pp.28-34, http://dx.doi.org/10.1109/MC.2007.225.

[7] S. Charoensiriwath and P. Srichaikul, "Constructing Thailand's National Anthropometrics Database using 3D Body Scanning Technology", in Proc. of the Pacific Neighborhood Consortium Conference, Taipei, Taiwan, 2009, http://www.pnclink.org/pnc2009/english/Abstract/08-Unfoldinge-Culture/08 UnfoldingeCulture Ab stract PiyawutSrichaikul.pdf., accessed 2014.

[8] N. Anand, 'Size' does matter. Need for 'Size India'- National Body Sizing Survey of Indian, http://www.techexchange.com/library/Size\%20does\%20matter\%20-\%20Need\%20for\%20Size\%2 Olndia\%20-\%20National\%20Body\%20Sizing\%20Survey\%20of\%20Indian.pdf., accessed 2014.

[9] M. R. Nelson et al, "Designing database to store biological information", in BIOSILICO, Vol.1, Issue 4, 2003, pp. 134-140, http://dx.doi.org/10.1016/S1478-5382(03)02357-6.

[10]G. Post \& A. Kagan, "Database management systems: design considerations and attribute facilities", in Journal of Systems and Software, Vol.56, Issue 2, 2001, pp. 183-193, http://dx.doi.org/10.1016/S0164-1212(00)00096-0.

[11] J. C. K. Wells, P. Treleaven and S. Charoensiriwath, "Body shape by 3-D photonic scanning in Thai and UK adults: comparison of national sizing surveys", in International Journal of Obesity, Vol.36, 2012, pp.148-154, http://dx.doi.org/10.1038/ijo.2011.51. 